\title{
Deconvolution of instrumental aberrations for synchrotron powder X-ray diffractometry
}

\section{T. Ida, H. Hibino and H. Toraya}


Journal of

Applied Crystallography

ISSN 0021-8898

Received 2 September 2002

Accepted 18 November 2002

(C) 2003 International Union of Crystallography Printed in Great Britain - all rights reserved

\section{Deconvolution of instrumental aberrations for synchrotron powder X-ray diffractometry}

\author{
T. Ida, ${ }^{*}$ H. Hibino and H. Toraya
}

Ceramics Research Laboratory, Nagoya Institute of Technology, Asahigaoka, Tajimi, Gifu 5070071, Japan. Correspondence e-mail: ida@crl.nitech.ac.jp

A method to remove the effects of instrumental aberrations from the whole powder diffraction pattern measured with a high-resolution synchrotron powder diffractometer is presented. Two types of asymmetry in the peak profiles caused by (i) the axial-divergence aberration of the diffractometer (diffractometer aberration) and (ii) the aberration of the monochromator and focusing optics on the beamline (beamline aberration) are both taken into account. The method is based on the whole-pattern deconvolution by Fourier technique combined with the abscissa-scale transformation appropriate for each instrumental aberration. The experimental powder diffraction data of $\mathrm{LaB}_{6}$ (NIST SRM660) measured on beamline BL- $4 \mathrm{~B}_{2}$ at the Photon Factory in Tsukuba have been analysed by the method. The formula of the scale transformation for the diffractometer aberration has a priori been derived from the instrumental function with geometric parameters of the optics. The strongly deformed experimental peak profiles at low diffraction angles have been transformed to sharp peak profiles with less asymmetry by the deconvolution of the diffractometer aberration. The peak profiles obtained by the deconvolution of the diffractometer aberration were modelled by an asymmetric model profile function synthesized by the convolution of the extended pseudo-Voigt function and an asymmetric component function with an empirical asymmetry parameter, which were linearly dependent on the diffraction angle. Fairly symmetric peak profiles have been obtained by further deconvolution of the empirically determined asymmetric component of the beamline aberration.

\section{Introduction}

Synchrotron radiation is a very powerful tool for the purpose of structure analysis by powder diffractometry, because the low divergence of the source X-ray beam provides fairly high resolution in powder diffraction data. The sharp and simple diffraction peak profiles of a well crystallized sample measured with a synchrotron light source enable precise estimation of lattice constants (Hart et al., 1990) and accurate structure determination (Estermann \& Gramlich, 1993). The sharp instrumental function also provides improved sensitivity in size-strain estimation by line broadening analysis (Cox, 1992).

It is strongly desired to establish the accurate formula of the instrumental function for further improvement in the peak profile analysis. Previously, we have developed a mathematical model for the instrumental function for a high-resolution synchrotron powder diffractometer equipped with a crystal analyser (Ida et al., 2001). However, it has been difficult to construct a model for the effects of the spectral distribution and beam divergence of the source X-ray on the diffraction peak profiles, because those effects are not only affected by many parameters concerning the monochromator and focusing optics on a beamline at a synchrotron facility, but are also mutually correlated in a complex manner owing to the high coherence of the light source.

Recently, we have proposed a novel Fourier-based method to eliminate the effects of instrumental aberrations from the whole powder diffraction pattern measured with a conventional Bragg-Brentano powder X-ray diffractometer (Ida \& Toraya, 2002). As the method is based on the analytical model for the effects of instrumental aberrations, the whole powder diffraction pattern can immediately be deconvoluted without the use of any standard materials.

In this study, we have applied the Fourier-based method to eliminate the effect of the axial-divergence aberration of a high-resolution synchrotron diffractometer (diffractometer aberration) from the experimental diffraction data of $\mathrm{LaB}_{6}$ powder measured on beamline BL-4B $\mathrm{B}_{2}$ at the Photon Factory in Tsukuba, and investigated the effects of the beamline optics (beamline aberration) on the peak profiles. We have also tried to remove the residual asymmetric feature caused by the beamline aberration, applying an empirical model for the asymmetry. 


\section{Diffractometer aberration}

In this section, the analytical formulae necessary for eliminating the diffractometer aberration from the entire diffraction data are described.

\subsection{Instrumental function of the diffractometer}

The axial-divergence effect limited by a set of Soller slits on the diffracted beam side is the only significant aberration of a high-resolution powder diffractometer (Ida et al., 2001). The instrumental function for the axial-divergence aberration is given by

$$
\omega_{A}(\Delta 2 \theta)= \begin{cases}\left(\beta \gamma_{A}\right)^{-1}\left[\left(-\Delta 2 \theta / \beta \gamma_{A}\right)^{-1 / 2}-1\right] \\ & \text { for }-\beta \gamma_{A}<\Delta 2 \theta<0 \\ 0 & \text { elsewhere }\end{cases}
$$

with

$$
\beta=\Phi^{2} / 2
$$

and

$$
\gamma_{A}=\cot 2 \theta+\tan \Theta_{A},
$$

for any diffraction angles $2 \theta$, where $\Phi$ is the full width at halfmaximum (FWHM) of the angular distribution of the axial divergence limited by the Soller slits, and $\Theta_{A}$ is the Bragg angle at the crystal analyser.

\subsection{Scale transform of the instrumental function}

Even though the width of the instrumental function $\omega_{A}(\Delta 2 \theta)$ depends on the diffraction angle $2 \theta$, an abscissaindependent formula can be derived by applying an appropriate scale transform (Ida \& Toraya, 2002). Here we apply the following scale-transform function $G_{A}(2 \theta)$,

$$
\begin{aligned}
\chi= & G_{A}(2 \theta) \\
= & {\left[2 \theta \sin \Theta_{A}-\cos \Theta_{A} \ln \left(\sin 2 \theta \tan \Theta_{A}+\cos 2 \theta\right)\right] } \\
& \times \cos \Theta_{A},
\end{aligned}
$$

in order to satisfy the following relation (Ida et al., 2001):

$$
\Delta \chi / \Delta 2 \theta=1 /\left(\cot 2 \theta+\tan \Theta_{A}\right)=1 / \gamma_{A} .
$$

When we define the instrumental function $w_{A}(\Delta \chi)$ for the transformed abscissa $\chi$ by

$$
w_{A}(\Delta \chi) \equiv \gamma_{A} \omega_{A}(\Delta 2 \theta)
$$

the abscissa-independent formula of the instrumental function is given by

$$
w_{A}(\Delta \chi)=\left\{\begin{array}{c}
\beta^{-1}\left[(-\Delta \chi / \beta)^{-1 / 2}-1\right] \\
\text { for }-\beta<\Delta \chi<0, \\
0 \text { elsewhere. }
\end{array}\right.
$$

Therefore, the intensity data multiplied by $\gamma_{A}$ accompanied with the transformed abscissa $\chi$ are considered as a convolution with the abscissa-independent instrumental function $w_{A}(\Delta \chi)$.

\subsection{Fourier transform of the instrumental function}

The Fourier transform of the instrumental function $w_{A}(\Delta \chi)$,

$$
W_{A}(\xi)=\int_{-\infty}^{\infty} w_{A}(x) \exp (2 \pi i \xi x) \mathrm{d} x
$$

is solved directly from equation (7). The solution is given by

$$
\begin{aligned}
W_{A}(\xi)= & (\beta \xi)^{-1 / 2}\left\{C\left[2(\beta \xi)^{1 / 2}\right]-i S\left[2(\beta \xi)^{1 / 2}\right]\right\} \\
& -(2 \pi i \beta \xi)^{-1}[1-\exp (-2 \pi i \beta \xi)],
\end{aligned}
$$

where $C(x)$ and $S(x)$ are the Fresnel functions defined by

$$
S(x) \equiv \int_{0}^{x} \sin \left(\pi t^{2} / 2\right) \mathrm{d} t
$$

and

$$
C(x) \equiv \int_{0}^{x} \cos \left(\pi t^{2} / 2\right) \mathrm{d} t .
$$

Reliable computer routines to evaluate Fresnel functions are available (Press et al., 1986).

\section{Method of data processing}

In this section, a series of actual procedures of the deconvolution are presented. Further details have been described elsewhere (Ida \& Toraya, 2002).

\subsection{Scale transform of data}

Since the polarization factor can be neglected in synchrotron diffractometry, only the corrections for the angular velocity and powder diffraction intensity factors (Lipson \& Langford, 1999) given by

$$
f_{\text {corr }}(2 \theta)=1 / \sin \theta \sin 2 \theta
$$

are taken into account.

First, the following scale transforms for the diffraction angle $\left\{2 \Theta_{m}\right\}$, intensity $\left\{S_{m}\right\}$ and error data $\left\{\Delta S_{m}\right\}(m=0, \ldots, M-1)$ are applied:

$$
\begin{gathered}
\chi_{m} \longleftarrow G_{A}\left(2 \Theta_{m}\right), \\
s_{m} \longleftarrow S_{m} \gamma_{A}\left(2 \Theta_{m}\right) / f_{\text {corr }}\left(2 \Theta_{m}\right), \\
\Delta s_{m} \longleftarrow \Delta S_{m} \gamma_{A}\left(2 \Theta_{m}\right) / f_{\text {corr }}\left(2 \Theta_{m}\right),
\end{gathered}
$$

where $\left\{\chi_{m}\right\}$ is the abscissa, $\left\{s_{m}\right\}$ the ordinate and $\left\{\Delta s_{m}\right\}$ the error data.

\subsection{Interpolation}

$N$-point equidistant data are created by interpolating $M$ point non-equidistant data on the transformed scale. In order to keep the statistical properties of the data unchanged by the interpolation, the error data are modified before the interpolation process by the following equation:

$$
\Delta s_{m}^{\prime} \longleftarrow\left[\frac{\left(2 \Theta_{m+1}-2 \Theta_{m-1}\right) N}{2\left(\chi_{M-1}-\chi_{0}\right) \gamma_{A}\left(2 \Theta_{m}\right)}\right]^{1 / 2} \Delta s_{m} .
$$

The equidistant data sets of abscissa $\left\{x_{n}\right\}$, ordinate $\left\{y_{n}\right\}$ and error $\left\{\Delta y_{n}\right\}(n=0, \ldots, N-1)$ are created by applying cubic spline interpolation to the sets of $\left\{\chi_{m}\right\},\left\{s_{m}\right\}$ and $\left\{\Delta s_{m}^{\prime}\right\}$. 


\subsection{Deconvolution by Fourier method}

As $\left\{y_{n}\right\}$ is a set of equidistantly spaced data, the Fourier transform $\left\{Y_{k}\right\}$,

$$
Y_{k}=\sum_{0}^{N-1} y_{n} \exp (2 \pi i k n / N) \quad(k=-N / 2, \ldots, N / 2),
$$

can be rapidly evaluated by fast Fourier transform (FFT).

The discrete Fourier transform $\left\{W_{k}\right\}$ of the instrumental function $w_{A}(x)$ is calculated by

$$
W_{k}=W_{A}(k / N \Delta x) \quad(k=-N / 2, \ldots, N / 2),
$$

where $\Delta x$ is the spacing of the data $\left\{y_{n}\right\}$, that is, $\Delta x=x_{n+1}-x_{n}$. Periodicities of $Y_{k}=Y_{N+k}$ and $W_{k}=W_{N+k}$ are assumed.

According to the convolution theorem, the deconvoluted data $\left\{z_{n}\right\}$ are given by

$$
z_{n}=N^{-1} \sum_{k=0}^{N-1}\left(Y_{k} / W_{k}\right) \exp (-2 \pi i k n / N)
$$

which are also calculated by FFT method.
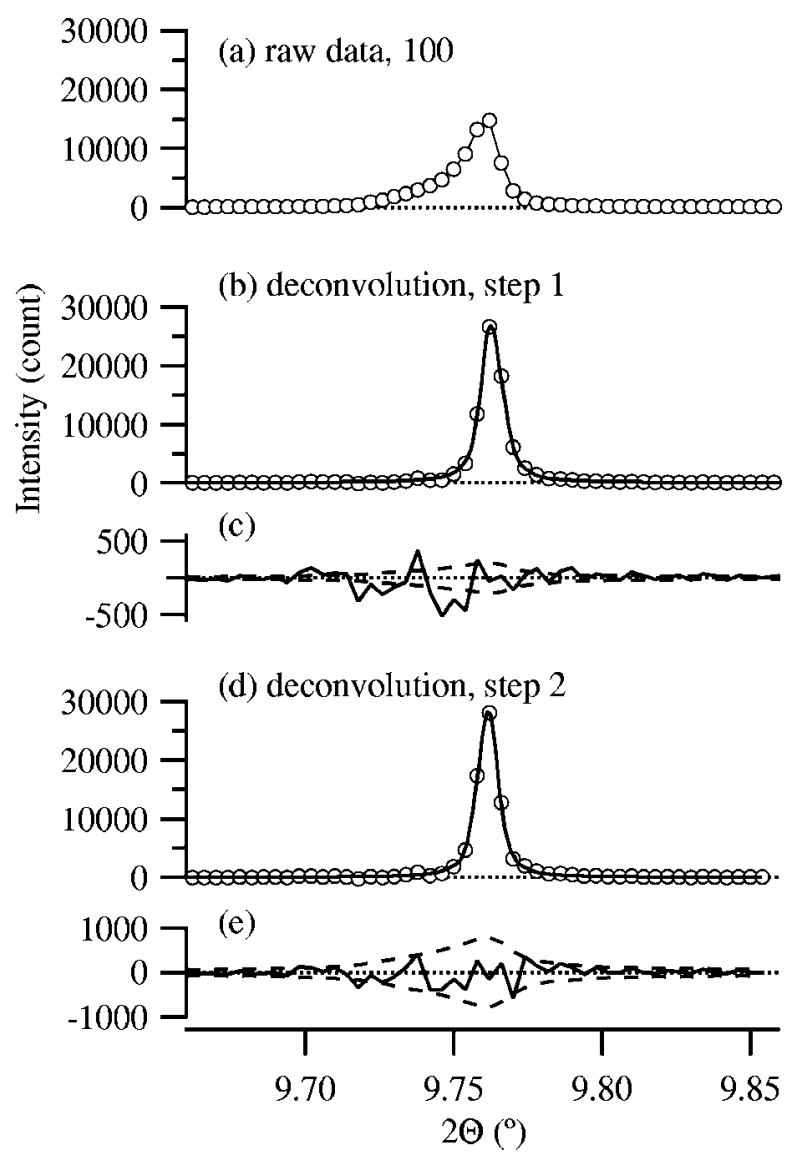

Figure 1

(a) The experimental peak profile of $\mathrm{LaB}_{6} 111$ reflection measured with a synchrotron X-ray $(\lambda=0.707 \AA$ ). (b) The data given by the deconvolution of the instrumental function of the diffractometer (open circles) and the optimized curve (solid line). (c) The errors in the deconvoluted data estimated by Fourier method (broken line) and difference (solid line) in the fitting shown in $(b) .(d)$ The data given by the deconvolution of the asymmetry caused by beamline optics (open circles) and the optimized curve (solid line). (e) The errors in the deconvoluted data (broken line) and difference (solid line) for the fitting shown in $(d)$.

\subsection{Error estimation by Fourier method}

The errors $\left\{\Delta z_{n}\right\}$ attached to the deconvoluted data $\left\{z_{n}\right\}$ are modelled by the reciprocal of the square root of the correlation between the reciprocal of the variance in the source data and the squared instrumental function (Ida \& Toraya, 2002), which are evaluated by the following equations:

$$
\begin{gathered}
\Delta z_{n}=\left\{N^{-1} \sum_{k=0}^{N-1} V_{k}^{(-2)}\left[W_{k}^{(2)}\right]^{*} \exp (-2 \pi i k n / N)\right\}^{-1 / 2}, \\
V_{k}^{(-2)}=\sum_{n=0}^{N-1}\left(\Delta y_{n}\right)^{-2} \exp (2 \pi i k n / N), \\
W_{k}^{(2)}=\sum_{n=0}^{N-1} w_{n}^{2} \exp (2 \pi i k n / N), \\
w_{n}=N^{-1} \sum_{k=0}^{N-1} W_{k} \exp (-2 \pi i k n / N),
\end{gathered}
$$

where $\left[W_{k}^{(2)}\right]^{*}$ denotes the complex conjugate of $W_{k}^{(2)}$.

\subsection{Inverse scale transformation and interpolation}

In order to make comparison of the deconvoluted and source data easier, the deconvoluted data are mapped onto the original scale again. Intensity $\left\{t_{m}\right\}$ and error $\left\{\Delta t_{m}^{\prime}\right\}$ data mapped onto the sampling points $\left\{\chi_{m}\right\}$ are created by cubic spline interpolation from the deconvoluted intensity $\left\{z_{n}\right\}$ and error $\left\{\Delta z_{n}\right\}$ data. The mapped error data $\left\{\Delta t_{m}^{\prime}\right\}$ are corrected by

$$
\Delta t_{m} \longleftarrow\left[\frac{\left(2 \Theta_{m+1}-2 \Theta_{m-1}\right) N}{2\left(\chi_{M-1}-\chi_{0}\right) \gamma_{A}\left(2 \Theta_{m}\right)}\right]^{-1 / 2} \Delta t_{m}^{\prime}
$$

to cancel the change in the statistical properties introduced by the interpolation. The final deconvoluted intensity $\left\{T_{m}\right\}$ and error $\left\{\Delta T_{m}\right\}$ data for the diffraction angles $\left\{2 \Theta_{m}\right\}$ are calculated by the following equations:

$$
T_{m} \longleftarrow t_{m} f_{\text {corr }}\left(2 \Theta_{m}\right) / \gamma_{A}\left(2 \Theta_{m}\right)
$$

and

$$
\Delta T_{m} \longleftarrow \Delta t_{m} f_{\text {corr }}\left(2 \Theta_{m}\right) / \gamma_{A}\left(2 \Theta_{m}\right)
$$

\section{Analysis of experimental data}

\subsection{Experimental}

The diffraction data of standard $\mathrm{LaB}_{6}$ powder [National Institute of Standards \& Technology, SRM660, $a=$ $4.15695(6) \AA]$ were collected with a high-resolution synchrotron powder diffractometer, MDS (Toraya et al., 1996), on beamline BL- $4 \mathrm{~B}_{2}$ at the Photon Factory (PF) in Tsukuba.

The PF storage ring stored $2.5 \mathrm{GeV}$ electron beams and was operated in multi-bunch mode. The beam radiated from a bending magnet is used on beamline $B L-4 B_{2}$. The white radiation is monochromated with an $\mathrm{Si}(111)$ flat doublecrystal monochromator and focused with a cylindrical mirror (1000 $\mathrm{mm}$ in length, $62.5 \mathrm{~mm}$ in curvature) onto the specimen 
centred at the diffractometer. Incident slits were inserted between the focusing mirror and the specimen. The monochromator, focusing mirror, incident slits and specimen were located at distances of about 17, 18, 28 and $29 \mathrm{~m}$ from the bending magnet, respectively. The use of the cylindrical focusing mirror and the rectangular cross section restricted by a couple of incident slits may cause asymmetric divergence and spectral broadening of the incident beam.

The $\mathrm{LaB}_{6}$ powder was loaded into a flat aluminium sample holder, which was mounted on an attachment, rotated at 1 revolution $\mathrm{s}^{-1}$ about the normal to the sample surface during the measurements. The sample face was inclined by $4.885^{\circ}$ to the incident beam. The diffractometer was equipped with a set of Soller slits, with an axial-divergence angle $\Phi=1^{\circ}$, and a Ge(111) crystal analyser $\left(d_{111}=3.26638 \AA\right)$ adjusted at $\Theta_{A}=$ $6.2^{\circ}$ for the wavelength $0.707 \AA$ on the diffracted-beam side. The incident X-ray beam was restricted to $2.5 \mathrm{~mm}$ in width and $1 \mathrm{~mm}$ in height with a couple of incident slits.

The diffraction pattern was scanned over the angular range $9.0-37.8^{\circ}(2 \theta)$, with a step length of $0.004^{\circ}(2 \theta)$ and a counting time of $4 \mathrm{~s} \mathrm{step}^{-1}$.

\subsection{Deconvolution of the diffractometer aberration}

The deconvolution by the method described in $\$ \S 2$ and 3 was applied to the experimental powder diffraction data. The
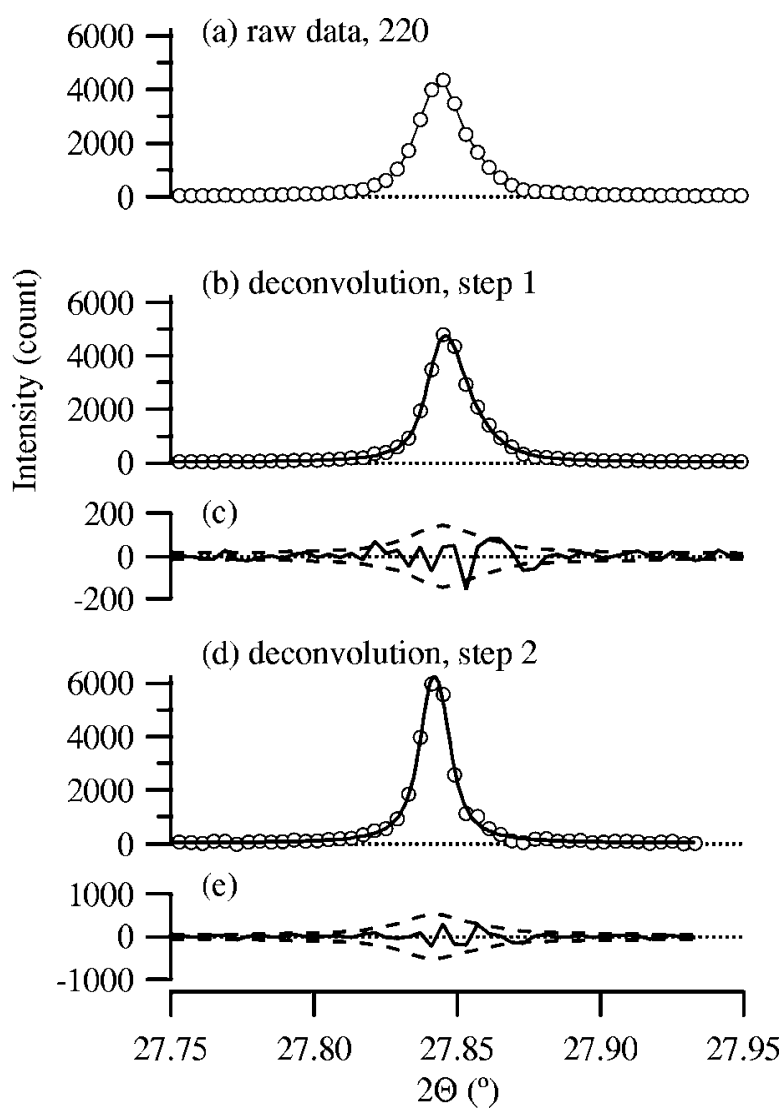

Figure 2

(a) The experimental peak profile of $\mathrm{LaB}_{6} 220$ reflection measured with a synchrotron X-ray $(\lambda=0.707 \AA)$. See the caption of Fig. 1 for $(b),(c),(d)$ and $(e)$. number of sampling points of the interpolated data was $N=$ 32768. Figs. 1-3(a) show the experimental peak profiles of 100, 220 and 321 reflections, and Figs. 1-3(b) show the data obtained by the deconvolution of the diffractometer aberration as open circles. Note that the whole diffraction pattern was simultaneously treated by the method, though only restricted regions are shown in Figs. 1-3.

As shown in Figs. 1(a) and 1(b), the heavily distorted peak profiles at low diffraction angles are transformed to sharp and almost symmetric profiles by the deconvolution of the instrumental function of the diffractometer, which indicates that the peak profiles at low diffraction angles are dominated by the axial-divergence aberration of the diffractometer.

In contrast, the peak profiles at higher diffraction angles show significant asymmetry, having longer tails on the higherangle side of the peak. The higher-angle peak profiles are only slightly changed by the deconvolution of the diffractometer aberration, as can be seen in Figs. 2(a), 2(b), 3(a) and 3(b). The residual asymmetry in the deconvoluted profiles is naturally ascribable to the beamline aberration.

\subsection{Evaluation of the beamline aberration}

It is difficult to deduce a mathematical model from the geometry of the beamline optics for the following reasons: (i)
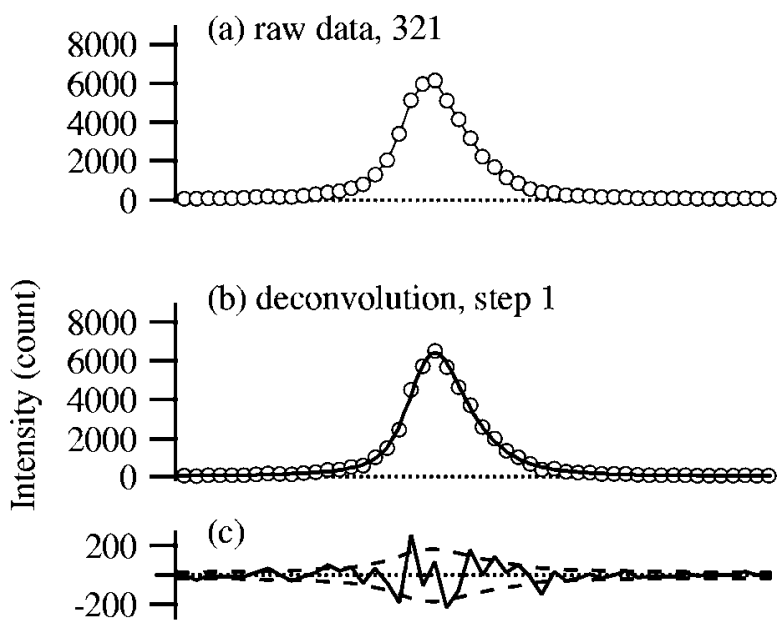

(d) deconvolution, step 2
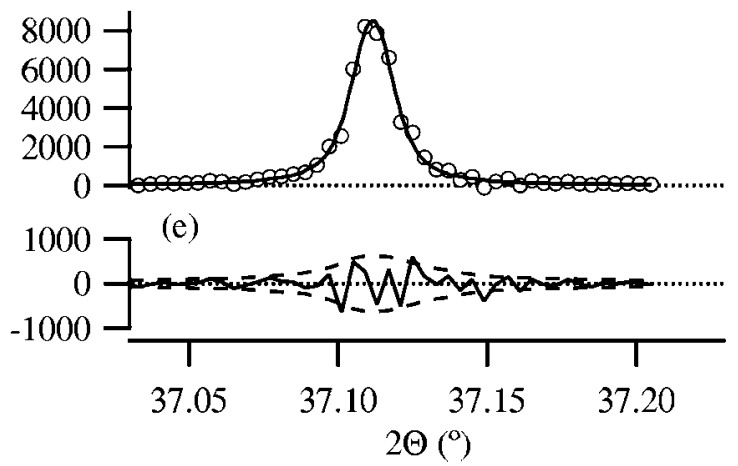

Figure 3

(a) The experimental peak profile of $\mathrm{LaB}_{6} 321$ reflection measured with a synchrotron X-ray $(\lambda=0.707 \AA)$. See the caption of Fig. 1 for $(b),(c),(d)$ and $(e)$. 
the effects of the monochromator and focusing mirror on the diffraction peak profiles are affected by many geometrical parameters of the optics; (ii) the spectral distribution and divergence of the source X-ray beam are heavily correlated; (iii) the wavelength, focusing condition and cross section of the X-ray beam are frequently changed; and (iv) the characteristics of the X-ray beam are slightly varied on each injection of the electrons to the storage ring.

In this section, we analyse the asymmetry caused by the beamline aberration in the experimental peak profiles extracted by the deconvolution of the diffractometer aberration. The dependence of the extracted asymmetry on the diffraction angle is estimated by a curve-fitting method applying an asymmetric model peak profile function. We use the model function synthesized by the convolution of the extended pseudo-Voigt function (Ida et al., 2000) with the following exponential function as an asymmetric component:

$$
\omega_{E}(\Delta 2 \theta)=\left\{\begin{array}{l}
\gamma_{E}^{-1} \exp \left(-\Delta 2 \theta / \gamma_{E}\right) \\
\text { for } 0<\Delta 2 \theta \\
0 \quad \text { elsewhere }
\end{array}\right.
$$

The convoluted model profile has been calculated by applying an efficient algorithm for the numerical integral (Ida, 1998; Ida \& Kimura, 1999). The extended pseudo-Voigt functions can be considered to be virtually identical to the Voigt function defined as the convolution of the Lorentzian and Gaussian functions (Ida et al., 2000). Only the parameter $\gamma_{E}$ in the asymmetric component function specifies the asymmetry of the profile caused by the beamline aberration.

The model profile function includes the peak position $2 \theta_{0}$, integrated intensity $I$, Lorentzian FWHM $\Gamma_{L}$, Gaussian FWHM $\Gamma_{G}$, asymmetry parameter $\gamma_{E}$, and constant background $b$ as adjustable parameters. The parameters are optimized by a least-squares method for the data obtained by the deconvolution of the diffractometer aberration. The peak profile analysis has been conducted for all the diffraction peaks in the measured range, from 100 to 321 reflections.

Figs. 1-3(b) show the optimized asymmetric model peak profiles for 100, 220 and 321 reflections as solid curves. The

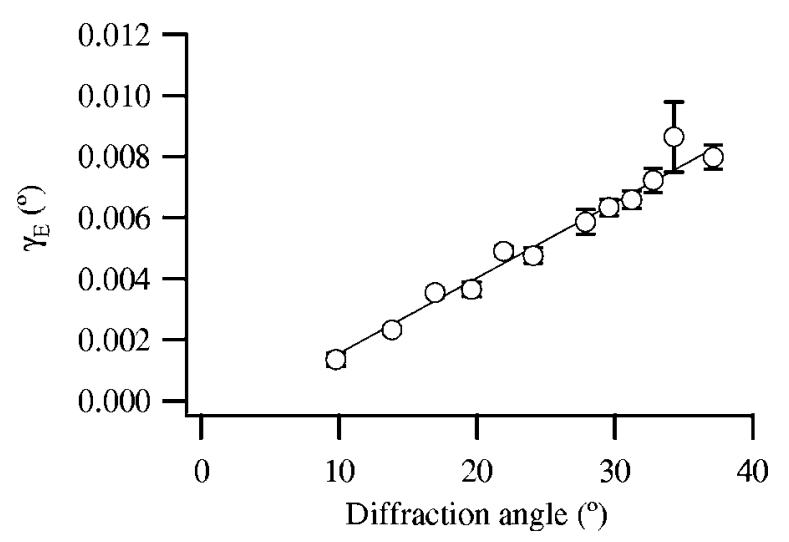

Figure 4

The dependence of the asymmetry parameter $\left(\gamma_{E}\right)$ for the beamline aberration upon the diffraction angle (open circles). Error bars indicate $1 \times$ the standard uncertainty. The optimized linear dependence is shown by the thin line. optimized profiles are very close to the data obtained by the deconvolution of the diffractometer aberrations (open circles). The differences are comparable with the errors estimated by the Fourier method, and no systematic behaviours are found in the difference, as shown in Figs. 1-3(c). We can conclude that the model applied here has been validated within the experimental errors, even though the geometric description of the beamline optics has not yet been established.

The dependence of the optimized asymmetry parameter $\gamma_{E}$ on the diffraction angle is shown in Fig. 4. The optimized linear dependence is given by

$$
\gamma_{E}=a+b(2 \theta), \quad a=-0.00093(19)^{\circ}, \quad b=0.000248(9) .
$$

The linear dependence on the diffraction angle $2 \theta$, rather than $\tan \theta$ or $1 / \cos \theta$, suggests that the asymmetry is caused by the instrumental aberrations of the beamline optics, which are considered to be mutually correlated effects of the spectral distribution and divergence of the source X-ray beam.

\subsection{Elimination of experimentally evaluated asymmetry}

Assuming the linear dependence of the asymmetry parameter $\gamma_{E}$ on the diffraction angle, the residual asymmetry can also be eliminated by the deconvolution based on the Fourier and scale-transform methods from the whole diffraction pattern.

We use the scale-transform function

$$
G_{E}(2 \theta)=(1 / b) \ln |2 \theta+a / b|
$$

in order to satisfy the relation

$$
\mathrm{d} G_{E}(2 \theta) / \mathrm{d}(2 \theta)=1 /[a+b(2 \theta)]=1 / \gamma_{E}(2 \theta) .
$$

The transformed instrumental function is given by

$$
w_{E}(x)= \begin{cases}\exp (-x) & \text { for } x>0, \\ 0 & \text { elsewhere }\end{cases}
$$

and the Fourier transform is

$$
W_{E}(\xi)=\int_{-\infty}^{\infty} w_{E}(x) \exp (2 \pi i \xi x) \mathrm{d} x=1 /(1-2 \pi i \xi) .
$$

The actual procedures for the deconvolution of the beamline aberration are similar to those described in $\S 3$, except that $G_{A}(2 \theta), \gamma_{A}(2 \theta)$ and $W_{A}(\xi)$ are substituted by $G_{E}(2 \theta), \gamma_{E}(2 \theta)$ and $W_{E}(\xi)$.

The results of the deconvolution are shown as open circles in Figs. 1-3 $(d)$. The sampling-point number of the interpolated data was $N=16384$. It has been found that sharpened and fairly symmetrized profiles are obtained by the deconvolution for all the diffraction peaks.

Significant peak shifts by the deconvolution are also observed in Figs. 1-3. This indicates that the experimental peak positions are shifted by the instrumental aberrations, and the deconvolution automatically corrects the systematic errors, which enables accurate determination of the intrinsic peak positions (Ida \& Kimura, 1999). 
Table 1

Optimized parameters for the symmetrized diffraction peak profiles.

\begin{tabular}{|c|c|c|c|c|c|c|}
\hline$h k l$ & $2 \theta_{0}\left({ }^{\circ}\right)$ & $I$ & $\Gamma_{L}\left(^{\circ}\right)$ & $\Gamma_{G}\left({ }^{\circ}\right)$ & $b$ & $R_{\mathrm{wP}}(\%)$ \\
\hline 100 & 9.7615 (1) & $316(9)$ & $0.0051(6)$ & $0.0049(5)$ & $40(17)$ & 4.0 \\
\hline 110 & 13.8185 (1) & 698 (14) & $0.0055(7)$ & $0.0061(5)$ & $74(28)$ & 4.1 \\
\hline 111 & 16.9439 (2) & 357 (11) & $0.0056(10)$ & $0.0071(7)$ & $41(21)$ & 3.5 \\
\hline 200 & $19.5888(2)$ & $202(8)$ & 0.0060 (14) & $0.0066(10)$ & 41 (18) & 5.1 \\
\hline 210 & 21.9257 (2) & 469 (13) & 0.0058 (11) & $0.0078(7)$ & $42(26)$ & 5.0 \\
\hline 211 & $24.0505(2)$ & $263(10)$ & 0.0075 (16) & $0.0082(11)$ & $37(21)$ & 8.4 \\
\hline 220 & 27.8421 & $112(7)$ & 0.0059 (29) & 0.0093 (17) & $18(15)$ & 5.7 \\
\hline $300 / 221$ & $29.5678(2)$ & 331 (12) & $0.0066(18)$ & $0.0100(11)$ & $33(25)$ & 7.2 \\
\hline 310 & 31.2061 (3) & $235(10)$ & $0.0080(18)$ & 0.0088 (13) & $35(22)$ & 6.1 \\
\hline 311 & 32.7703 (4) & $156(8)$ & $0.0056(31)$ & 0.0110 (17) & 18 (19) & 7.8 \\
\hline 222 & 34.2707 (11) & $27(4)$ & 0.0059 (107) & $0.0127(57)$ & 32 (14) & 14.0 \\
\hline 320 & $43.5200(9)$ & $39(5)$ & $0.0083(75)$ & $0.0122(49)$ & $23(14)$ & 15.6 \\
\hline 321 & $37.1116(4)$ & $201(10)$ & $0.0098(26)$ & 0.0112 (19) & $27(23)$ & 9.0 \\
\hline
\end{tabular}

\subsection{Analysis of symmetrized peak profiles}

The symmetrized diffraction peak profiles obtained by the double deconvolutions have been analysed by a curve-fitting method with the extended pseudo-Voigt function (Ida et al., 2000) as a profile model. The model profile function includes the peak position $2 \theta_{0}$, integrated intensity $I$, Lorentzian FWHM $\Gamma_{L}$, Gaussian FWHM $\Gamma_{G}$, and constant background $b$ as adjustable fitting parameters.

The optimized values of the profile parameters are listed in Table 1. All the optimized values seem to be reasonable except that considerably larger errors are estimated as compared with typical results of profile analysis for raw diffraction data.

The reliability factor $R_{\mathrm{wP}}$, defined by the following equation, is also listed in Table 1.

$$
R_{\mathrm{wP}} \equiv\left\{\frac{\sum_{m}\left[T_{m}-T\left(2 \theta_{m}\right)_{\mathrm{calc}}\right]^{2} /\left(\Delta T_{m}\right)^{2}}{\sum_{m} T_{m}^{2} /\left(\Delta T_{m}\right)^{2}}\right\}^{1 / 2},
$$

where $\left\{T_{m}\right\}$ and $\left\{\Delta T_{m}\right\}$ are the deconvoluted intensity data and error data estimated by the Fourier method, respectively, and $T\left(2 \theta_{m}\right)_{\text {calc }}$ is the intensity value calculated with the optimized symmetric model function.

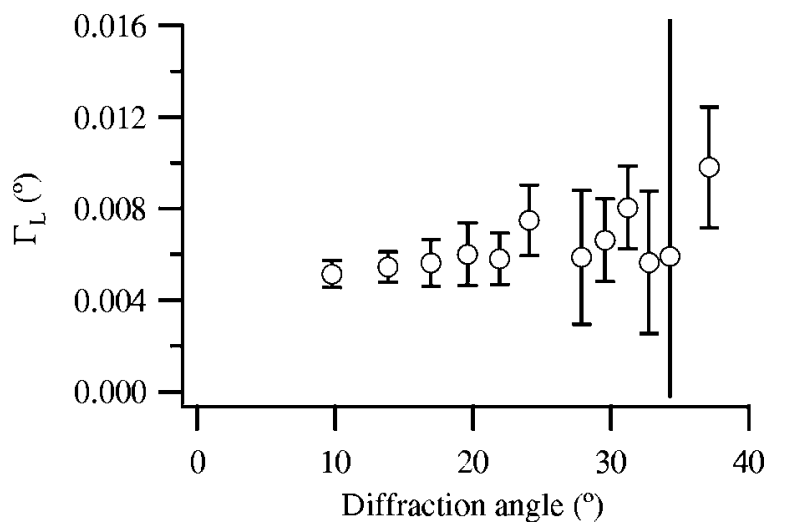

Figure 5

The dependence of the Lorentzian width $\left(\Gamma_{L}\right)$ on the diffraction angle. Error bars indicate $1 \times$ the standard uncertainty. The error bar for the 222 reflection $\left(34.27^{\circ}\right)$ is off the scale.
The optimized model profiles for 100, 220 and 321 reflections are shown as solid curves in Figs. 1-3(d). The errors estimated by the Fourier method in the symmetrized profiles are shown as broken lines, and the difference plots for the symmetric model are drawn as solid lines in Figs. 1-3(e). The differences are comparable with the estimated errors, and show no systematic behaviour.

The dependences of the optimized Lorentzian FWHM $\Gamma_{L}$ and Gaussian FWHM $\Gamma_{G}$, obtained by the profile-fitting method, on the diffraction angle are shown in Figs. 5 and 6. The values and dependences of those parameters are ascribable to (i) the intrinsic size and strain broadening and (ii) the finite resolution of the beamline optics.

Although it has been suggested that SRM660 LaB 6 shows small strain broadenings (Rasberry, 1989), quantitative descriptions of the broadenings have not been reported. The plots in Figs. 4 and 5 indicate that finite values of about $0.002-$ $0.005^{\circ}$ remain at the diffraction angle $0^{\circ}$ for both Lorentzian and Gaussian FWHM values. As the wavelength of the X-ray is $0.707 \AA$, those angular widths correspond to the coherence length of about $1 \mu \mathrm{m}$, which is smaller than the typical particle size of $10 \mu \mathrm{m}$ of the $\mathrm{LaB}_{6}$ (SRM660) powder (Rasberry, 1989). The symmetric component of the experimental profile should

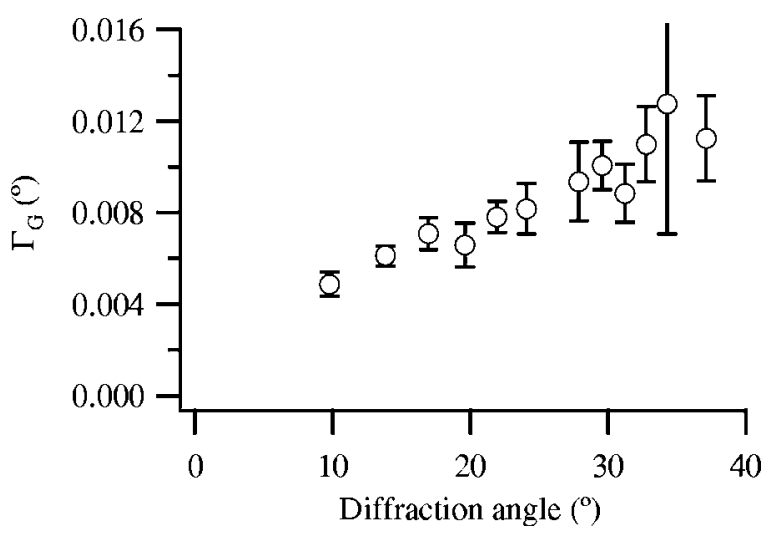

Figure 6

The dependence of the Gaussian width $\left(\Gamma_{G}\right)$ on the diffraction angle. Error bars indicate $1 \times$ the standard uncertainty. 
be attributed not only to the intrinsic broadening of the sample, but also to the finite resolution of the beamline optics.

The symmetric instrumental broadenings could be experimentally determined for any given arrangements of the beamline optics, if a standard material with well defined intrinsic peak profiles were available. Highly sensitive peak profile analysis will be achieved by correction or further deconvolution of the symmetric instrumental broadenings.

\section{Conclusion}

We have developed a deconvolution method to remove the diffractometer aberration and the asymmetry caused by the beamline optics from the whole powder diffraction pattern measured with a high-resolution synchrotron diffractometer equipped with a crystal analyser. The fairly symmetric peak profiles obtained by the deconvolution are well approximated by the Voigt profile, and the errors in the deconvoluted data are also reasonably estimated by a Fourier-based method. The deconvolution method provides automatic correction of the systematic errors in the peak position to improve the accuracy in the lattice-parameter determination. As the elimination of the asymmetry by the deconvolution certifies the validity of using symmetric model profile functions, the efficiency of structure analysis based on the Rietveld method will be enhanced. Highly sensitive peak profile analysis will immediately be achieved, if a well defined standard material for the intrinsic peak profiles is available.

\section{References}

Cox, D. E. (1992). Synchrotron Radiation Crystallography, pp. 186254. New York: Academic Press.

Estermann, M. A. \& Gramlich, V. (1993). J. Appl. Cryst. 26, 396-404. Hart, M., Cernik, R. J., Parrish, W. \& Toraya, H. (1990). J. Appl. Cryst. 23, 286-291.

Ida, T. (1998). Rev. Sci. Instrum. 69, 3837-3839.

Ida, T., Ando, M. \& Toraya, H. (2000). J. Appl. Cryst. 33, 1311-1316. Ida, T., Hibino, H. \& Toraya, H. (2001). J. Appl. Cryst. 34, 144-151. Ida, T. \& Kimura, K. (1999). J. Appl. Cryst. 32, 982-991. Ida, T. \& Toraya, H. (2002). J. Appl. Cryst. 35, 58-68.

Lipson, H. \& Langford, J. I. (1999). International Tables for Crystallography, Vol. C, edited by A. J. C. Wilson \& E. Prince, pp. 590-592. Dordrecht: Kluwer Academic Publishers.

Press, W. H., Flannery, B. P., Teukolsky, S. A. \& Vetterling, W. T. (1986). Numerical Recipes. Cambridge University Press.

Rasberry, S. D. (1989). Certificate of Analysis, Standard Reference Material 660. Instrument Line Position and Profile Shape Standard for X-ray Powder Diffraction. National Institute of Standards and Technology, Gaithersburg, MD, USA.

Toraya, H., Hibino, H. \& Ohsumi, K. (1996). J. Synchrotron Rad. 3, 75-83. 